\title{
Implementação de uma Ferramenta de Gerenciamento de Rotina e produtividade
}

\author{
Cíntia Cristina Bráz ${ }^{1}$; Andressa Pacífico Franco Quevedo ${ }^{2}$; Hesler Piedade Caffé Filho
}

\begin{abstract}
Resumo: A Gestão por Processos é uma abordagem focada na complexidade da estrutura gerencial para identificar e mapear seus processos e permitir um planejamento eficiente das atividades, a definição de responsabilidades e o uso eficaz dos recursos disponíveis levando a uma melhoria contínua do desempenho organizacional e dos resultados de negócios. Este artigo aborda o processo de implementação de uma ferramenta de gerenciamento de rotina e produtividade no Processo de Arrecadação do Escritório Local da Empresa Baiana de Águas e Saneamento S.A. (Embasa) em Paulo Afonso e tem como objetivo descrever o processo de implementação, bem como os benefícios agregados à gestão das atividades e seu reflexo na melhoria contínua dos resultados. A metodologia aplicada foi o Estudo de Caso com coleta de dados documental através de relatórios corporativos e análise descritiva. A Embasa, concessionária responsável pela prestação dos serviços de abastecimento de água e esgotamento sanitário de vários municípios da Bahia, identificou a necessidade de melhoria na gestão e, ao incorporar a Gestão por Processos, implementou ferramentas que proporcionaram a gestão e visibilidade das atividades dos processos, conseguindo elevar sua produtividade e obter melhores resultados.
\end{abstract}

Palavras-Chave: Gestão por Processos. Ferramenta. Resultado.

\section{Implementing a routine and productivity Management Tool}

\begin{abstract}
Process Management is an approach focused on the complexity of the management structure to identify and map their processes and enable efficient planning of activities, the definition of responsibilities and the effective use of available resources leading to a continuous improvement of organizational performance and business results. This article discusses the process of implementing a routine and productivity management tool in Local Office Aquisition Process of the Bahia Company of Water and Sanitation SA (Embasa) in Paulo Afonso and aims to describe the implementation process and the benefits added to the management of activities and its reflection in the continuous improvement of results. The methodology used was the case study with data collection document through corporate reports, and descriptive analysis. The Embasa, the concessionaire responsible for the provision of water supply and sewage from several municipalities of Bahia, identified the need for improved management and to incorporate Process Management, has implemented tools that provided the management and visibility of process activities, managing to raise their productivity and achieve better results.
\end{abstract}

Keywords: Business Process Management. Tool. Result

\footnotetext{
${ }^{1}$ Pós-graduanda em Gestão Pública pela Universidade Federal do Vale do São Francisco (UNIVASF). Especialista em Governança de TI pelo Centro Universitário Leonardo Da Vinci (UNIASSELVI). Graduada em Pedagogia pela Universidade do Estado da Bahia (UNEB). E-mail: cinysan@hotmail.com.

${ }^{2}$ Mestre em Administração pela Universidade Federal de Pernambuco (UFPE). Professora Assistente da Universidade Federal Rural de Pernambuco. Especialista em Gestão da Capacidade Humana nas Organizações pela Universidade de Pernambuco (UPE). Graduada em Turismo pela Universidade Federal de Pernambuco (UFPE). E-mail: andressadm@ gmail.com.

${ }^{3}$ Bacharel em Administração Geral pelo Centro Universitário da Bahia e Mestre em Gestão de Políticas Públicas da Universidade Federal do Recôncavo da Bahia. Especialista em Marketing Institucional pela Faculdade São Francisco de Juazeiro e MBA em Gestão Estratégica de Negócios pela Escola de Engenharia Eletromecânica da Bahia. E-mail: hesler.caffe@univasf.edu.br
} 


\section{Introdução}

A Gestão por Processos visa integrar as estratégias e objetivos das organizações com as expectativas e necessidades dos seus clientes através de uma visão global dos processos com foco na melhoria contínua dos resultados.

Segundo Capote (2011), Gestão de Processos de Negócio é uma abordagem disciplinar para identificar, desenhar, executar, documentar, medir, monitorar, controlar e melhorar processos de negócios automatizados ou não, para alcançar resultados consistentes e alinhados com os objetivos estratégicos da organização.

A abordagem por processos permite melhor especificação do trabalho realizado, o desenvolvimento de sistemas, a gestão do conhecimento, o redesenho e a melhoria, por meio da análise do trabalho realizado de modo a identificar oportunidades de aperfeiçoamento (BRASIL: 2013, p. 13).

O processo de melhoria na gestão precisa envolver os diversos níveis de liderança na condução e implementação de iniciativas de otimização dos processos. Por sua vez, o gerenciamento da rotina é uma ferramenta complementar nesse processo, constituindo-se numa prática necessária à gestão de processos na medida em que viabiliza o monitoramento e controle, ou seja, a gestão e visibilidade das atividades dos processos com foco na melhoria contínua dos resultados.

Monitoramento e Controle: essencial para avaliar e garantir o alinhamento dos processos com os objetivos da organização. O principal objetivo desta fase é identificar a conformidade do que foi implementado, se as metas estabelecidas foram atingidas e a eficiência do processo foi maximizada, além de padronizar e implementar a melhoria contínua; monitorar a performance, riscos e conformidade; comunicar e disseminar informações da gestão por processos (BRASIL: 2013, p. 54).

Nesse contexto, este estudo versará sobre a implementação de uma ferramenta de gerenciamento de rotina e produtividade no Processo de Arrecadação do Escritório Local da Embasa em Paulo Afonso e tem como objetivo descrever o processo de implementação, bem como os benefícios agregados à gestão das atividades e seu reflexo na melhoria contínua dos resultados. Para tanto, a metodologia aplicada foi o Estudo de Caso com coleta de dados documental através de relatórios corporativos e análise descritiva.

\section{Gestão por Processos no Setor Público}

Em âmbito nacional a implementação da Gestão por Processos está referenciada por diversas 
iniciativas do Governo Federal em direção a excelência em gestão pública.

O Programa Nacional de Gestão Pública e Desburocratização (GesPública), instituído pelo Decreto 5.378 de 23/02/2005 é o resultado da evolução histórica dessas iniciativas e visa contribuir para a qualidade dos serviços públicos prestados ao cidadão e para o aumento da competitividade do Brasil.

Proveniente do GesPública - política pública fundamentada em um modelo de gestão específico - o Modelo de Excelência em Gestão Pública (MEGP) - sistema de gestão de referência para as organizações do setor público brasileiro - contém diretrizes expressas em seus critérios de excelência gerencial, técnicas e tecnologias para sua aplicação e práticas de gestão implantadas com sucesso.

Um dos instrumentos do MEGP é o Guia de Gestão de Processos que contempla orientações metodológicas a serem utilizadas pelos agentes de processos durante as atividades de construção e melhoria de modelos de processos e na contratação de serviços de modelagem de processos.

A importância da gestão de processos nos organismos públicos justifica-se pelo direcionamento à obtenção de resultados mediante o "foco no cidadão", proposição da Carta de Serviços ao Cidadão - instrumento que tornou-se obrigatório para os órgãos do Poder Executivo Federal que prestam atendimento direto ao público a partir da publicação do Decreto 6.932 de $11 / 08 / 2009$

Os processos representam um instrumento que permite aproximar as diretrizes estratégicas daqueles que executam o trabalho nas instituições públicas, permitindo o alcance de objetivos. O "foco no cidadão" faz com que o Setor Público oriente seus processos ao atendimento das necessidades deste agente e os prestadores de serviço do Governo devem ter seus processos modelados, automatizados e geridos, provendo maior controle e qualidade às iniciativas desempenhadas (BRASIL: 2011, p.6).

\section{Gerenciamento de Rotina e Produtividade}

Segundo Paim et al (2009), todas as organizações precisam coordenar seu modo de trabalho, contemplando, necessariamente, a forma como os recursos e tarefas são distribuídos e planejados, como as atividades são realizadas e monitoradas no dia-a-dia e como a organização promove as melhorias nas operações e na forma de coordenação do trabalho em si.

Para isso, as organizações têm na gestão de processos uma boa alternativa para o gerenciamento das suas rotinas com vistas a uniformizar as formas de trabalho, melhorar o fluxo de informações, reduzir tempo e custos de produção, aumentar a produtividade e o valor agregado dos processos, produtos e serviços promovendo, dessa forma, a elevação do patamar de seu desempenho e 
qualidade de suas entregas.

A utilização do gerenciamento da rotina como ferramenta da gestão pela qualidade promove uma organização dos processos, bem como estabelece a tomada de decisão baseada nos fatos identificados por meio de um controle de processos, capacitando a gerência para uma visão de futuro das metas a serem atingidas (SANTOS, LIMA e ABRANTES, 2006).

No gerenciamento da rotina das atividades que compõem um processo é fundamental alinhar a definição dos parâmetros a serem utilizados na condução de tais atividades à estratégia organizacional e conduzi-la de maneira a garantir a obtenção de qualidade no serviço. Segundo Campos (1992, p. 14), "um produto ou serviço de qualidade é aquele que atende perfeitamente, de forma confiável, de forma acessível, de forma segura e no tempo certo às necessidades do cliente".

Moura (1997, p. 30) define gerenciamento da rotina como a "ação de coordenar e executar as atividades do dia-a-dia em nível operacional, praticando o ciclo PDCA (Planejar, Desenvolver, Checar e Agir) e executando as atividades de acordo com as políticas e diretrizes da empresa". Ou seja, o gerenciamento da rotina envolve um conjunto de atividades e procedimentos executados de forma contínua e, para desenvolvê-lo, é necessário alinhar metas de desempenho e resultados, executar os padrões definidos para o processo, medir e avaliar o desempenho e os resultados gerados bem como divulgá-los, e implementar as melhorias que possam corrigir os desvios identificados e promover melhorias no processo.

\section{A Empresa Baiana de Águas e Saneamento S.A}

A Embasa é uma sociedade de economia mista responsável pela prestação dos serviços de abastecimento de água e esgotamento sanitário do estado da Bahia. Criada em 11 de maio de 1971, é administrada sob os princípios da descentralização geográfica, a qual ocorre por meio de dezenove unidades regionais distribuídas no interior e na região metropolitana de Salvador. A Embasa tem como missão garantir o acesso universalizado aos seus serviços de modo sustentável, contribuindo para a melhoria da qualidade de vida e o desenvolvimento do Estado, visando assim alcançar o ranking das três empresas do Brasil que mais avançaram na universalização dos serviços de água e esgotamento sanitário de modo sustentável.

Em meados de 2013 a Embasa contratou uma empresa de consultoria para desenvolver o Projeto Transforma objetivando implementar a Gestão por Processos como meio para integrar a estratégia organizacional com a operação e as necessidades dos clientes e partes interessadas, promovendo o desenvolvimento de uma cultura organizacional orientada à colaboração entre as áreas, 
Id on Line Revista Multidisciplinar e de Psicologia

Id on Line Multidisciplinary and Psychology Journal

aprimoramento dos processos críticos, aumento de maturidade na gestão, melhoria contínua nos resultados, visibilidade gerencial e adequação dos recursos através da estrutura e dimensão, a partir de ferramentas e práticas adequadas ao caso.

\section{Gestão por Processos na Unidade Regional de Paulo Afonso}

A Unidade Regional da Embasa em Paulo Afonso é responsável pela prestação dos serviços de abastecimento de água e esgotamento sanitário para 66.873 ligações/usuários, sendo composta por cinco Divisões (Divisão Administrativa, Divisão de Manutenção, Divisão de Esgotamento Sanitário, Divisão Operacional e Divisão Comercial) e mais quatro Escritórios Locais (Abaré, Canudos, Jeremoabo e Paulo Afonso).

\section{Processos do Escritório Local (EL) de Paulo Afonso}

O EL de Paulo Afonso é composto por cinco processos (Distribuição, Faturamento, Arrecadação, Serviços e Atendimento ao Público) responsáveis pelas atividades práticas e administrativas envolvidas na prestação dos serviços fins da Embasa na cidade de Paulo Afonso e povoados circunvizinhos.

O Processo de Arrecadação, foco deste estudo, objetiva o alcance das metas de cobrança e arrecadação do EL de Paulo Afonso, seguindo, para isso, a Régua de Cobrança definida no Manual de Cobrança de Débitos que é um instrumento normativo que abrange todos os requisitos e procedimentos estabelecidos pela Embasa para cobrança de débitos de usuários inadimplentes.

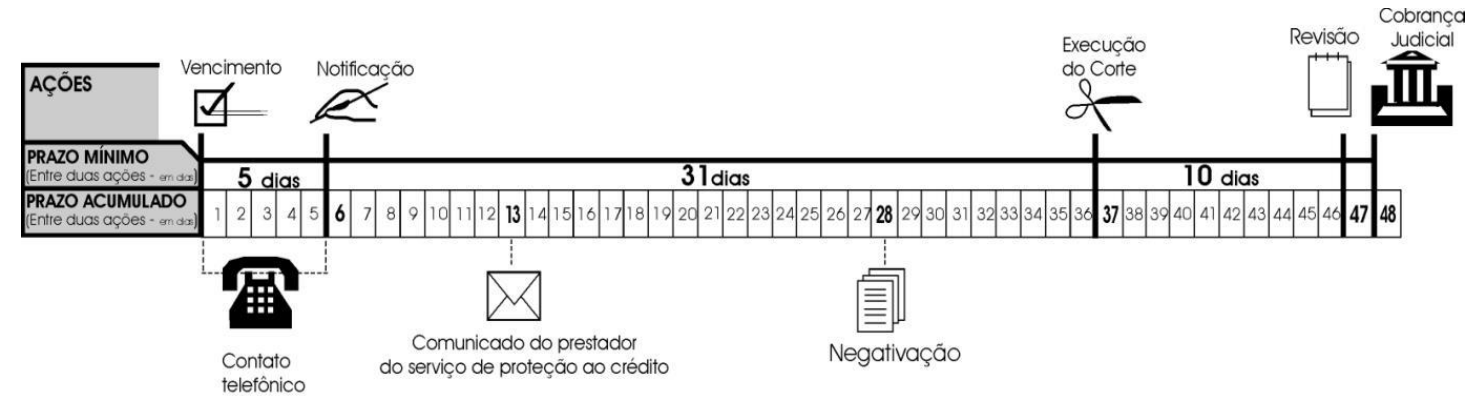

\begin{tabular}{|l|c|c|}
\hline Ações de Cobrança & Data & $\begin{array}{c}\text { Dias após o } \\
\text { vencimento }\end{array}$ \\
\hline Vencimento & $31 / 12$ & - \\
\hline Contato Telefônico & $01 / 01$ a 05/01 & \\
\hline Notificação & $06 / 01$ & 6 \\
\hline Comunicado & $13 / 01$ & 13 \\
\hline Negativação & $28 / 01$ & 28 \\
\hline Execução de Corte & $06 / 02$ & 37 \\
\hline Revisão & $17 / 02$ & 47 \\
\hline Cobrança Judicial & $18 / 02$ & 48 \\
\hline
\end{tabular}


Id on Line Revista Multidisciplinar e de Psicologia

Id on Line Multidisciplinary and Psychology Journal

Figura 01: Representação Gráfica da Régua de Cobrança.

Fonte: Manual de Cobrança de Débitos, p. 16.

A partir da Régua de Cobrança é possível dimensionar o ciclo do processo de cobrança e definir suas atividades, detalhando o fluxo de trabalho de acordo com a equipe disponível.

Materiais e Métodos

A metodologia aplicada nesse estudo foi a pesquisa qualitativa baseada num estudo descritivo.

Goldenberg (2003) justifica a aplicação da pesquisa qualitativa para a compreensão de fenômenos sociais no fato das abordagens qualitativas não se preocuparem em fixar leis para se produzir generalizações e se apoiarem no pressuposto da maior relevância do aspecto subjetivo da ação social, e sendo assim, contrapõem-se à incapacidade da estatística de dar conta de fenômenos complexos e da singularidade dos fenômenos que não podem ser identificados através de questionários padronizados.

O estudo descritivo serviu como base para descrever o fenômeno estudado. Segundo Best (1972, p.12-13), esse estudo "delineia o que é" - aborda também quatro aspectos: descrição, registro, análise e interpretação de fenômenos atuais, objetivando o seu funcionamento presente. Para Selltiz (1965, p.61-62), esse tipo de estudo "descreve um fenômeno ou situação mediante um estudo realizado em determinado espaço-tempo".

O procedimento metodológico utilizado foi baseado em um estudo de caso. Segundo Barros (2002, p.84), "nas ciências sociais este método caracteriza-se como uma metodologia de estudo que se volta à coleta de informações sobre um ou vários casos particulares".

Este método supõe que se pode adquirir conhecimento do fenômeno estudado a partir da exploração intensa de um ou mais casos específicos.

De acordo com Goldenberg (2003, p.33),

O estudo de caso reúne o maior número de informações detalhadas, por meio de diferentes técnicas de pesquisa, com o objetivo de apreender a totalidade de uma situação e descrever a complexidade de um caso concreto. Através de mergulho profundo e exaustivo em um objeto delimitado, o estudo de caso possibilita a penetração na realidade social, não conseguida pela análise estatística.

Para o levantamento dos dados foram utilizados como procedimentos a pesquisa bibliográfica e pesquisa documental.

A pesquisa bibliográfica é um resumo geral sobre os principais estudos publicados a respeito do objeto pesquisado, tornando-se assim de fundamental importância para o pesquisador por proporcionar uma visão sobre o quadro atual em que se encontram os estudos acerca do objeto em questão. 
Id on Line Revista Multidisciplinar e de Psicologia

Id on Line Multidisciplinary and Psychology Journal

De acordo com Barros (2002, p.34), "a pesquisa bibliográfica procura adquirir conhecimentos sobre um objeto de pesquisa a partir da busca de informações provindas de material gráfico, sonoro e informatizado". Para Lakatos (1986, p.57), "pesquisa bibliográfica é o levantamento de toda a bibliografia já publicada e que tenha relação com o tema estudado".

A pesquisa documental recorre a materiais que ainda não receberam tratamento analítico, ou seja, as fontes primárias. Nesse caso, foram utilizados os relatórios corporativos de Gestão, Administração e Demonstrações Financeiras da empresa estudada.

Após a manipulação dos dados e obtenção dos resultados, é necessário analisar e interpretar os mesmos, o que proporcionará respostas às investigações.

De acordo com Barros (2002, p.87),

[...] analisar significa buscar sentido mais explicativo dos resultados da pesquisa e a interpretação seria a capacidade de se voltar à síntese sobre os dados, entendendoos em relação a um todo maior, e em relação a outros estudos já realizados na mesma área.

Os dados coletados neste estudo foram analisados de forma qualitativa que caracteriza-se por apresentar os dados de forma verbal ou oral ou em forma de discurso, baseando-se na Dialética como linha filosófica, a qual exige que se estude cada fenômeno sob todos os aspectos, através do seu desenvolvimento. Foi utilizada a análise descritiva que delineou o fenômeno estudado.

Para Lakatos (1986, p.37),

[...] quando se deseja colher informações sobre aspectos de um grupo grande ou numeroso, verifica-se ser praticamente impossível fazer um levantamento do todo, o que acarreta na investigação apenas de uma parte dessa população ou universo, a qual deverá ser a mais representativa possível do todo.

Análise e Discussão dos Resultados

A partir de dados coletados nos relatórios corporativos de Gestão, Administração e Demonstrações Financeiras, foi possível fazer uma análise descritiva do processo de implantação da ferramenta de gerenciamento de rotina e produtividade no Processo de Arrecadação do Escritório Local da Embasa.

A necessidade de implementar essa ferramenta surgiu em 2013 a partir da análise das atividades demandadas no Processo de Arrecadação que exigem planejamento, monitoramento e controle, tornando necessário uma plataforma de gerenciamento que auxiliasse na otimização do Processo e consequente alcance dos resultados almejados.

Para tanto, foram aplicadas as etapas de Planejamento; Mapeamento do Processo; Redesenho 
Id on Line Revista Multidisciplinar e de Psicologia

Id on Line Multidisciplinary and Psychology Journal

da Rotina; Elaboração da Ferramenta; Monitoramento e Controle do Processo.

$\mathrm{Na}$ fase de planejamento foram definidos o material e os métodos necessários para o desenvolvimento e implantação da ferramenta, tais como:

- Material:

Ferramenta em formato XLS (Microsoft Office Excel).

- $\quad$ Métodos:

$\checkmark \quad$ Programação diária.

$\checkmark \quad$ Estratificação de Ordens de Serviço por categoria:

- Notificação

- Cobrança (pré-corte)

- Corte

- $\quad$ Revisão de corte

- Religação

- $\quad$ Revisão de inativas

$\checkmark \quad$ Estratificação de Resultados:

- Quantidade enviada

- Quantidade reprogramada

- Quantidade executada

- Quantidade pagas

- Sobras

$\mathrm{Na}$ fase de mapeamento foram definidas as responsabilidades dos agentes (colaboradores) envolvidos no processo e as atividades internas (administrativas) e externas (em campo), tais como:

- Atividades internas - 01 líder de processo e 02 colaboradoras:

$\checkmark \quad$ Coordenação da rotina: programar e controlar a rotina de serviços utilizando a ferramenta.

$\checkmark \quad$ Carteira de corte: controlar e encerrar ordens de corte no sistema comercial.

$\checkmark \quad$ Carteira de religação: controlar e encerrar ordens de religação no sistema comercial.

- $\quad$ Atividades externas -06 colaboradores:

$\checkmark \quad$ Entrega de notificação.

$\checkmark \quad$ Execução de cobrança (pré-corte). 
Id on Line Revista Multidisciplinar e de Psicologia

Id on Line Multidisciplinary and Psychology Journal

$$
\begin{array}{ll}
\checkmark & \text { Execução de corte. } \\
\checkmark & \text { Execução de revisão de corte. } \\
\checkmark & \text { Execução de religação. } \\
\checkmark & \text { Execução de revisão de inativas. }
\end{array}
$$

O redesenho da rotina foi desenvolvido para adequar a equipe à demanda, sendo tal fase constante no fluxo do processo, modificando-se de acordo com os resultados apresentados mensalmente.

A ferramenta foi elaborada na plataforma do Microsoft Office Excel, sendo utilizada uma planilha para cada mês, disponibilizando as funcionalidades de:

- $\quad$ Programar as atividades da equipe:

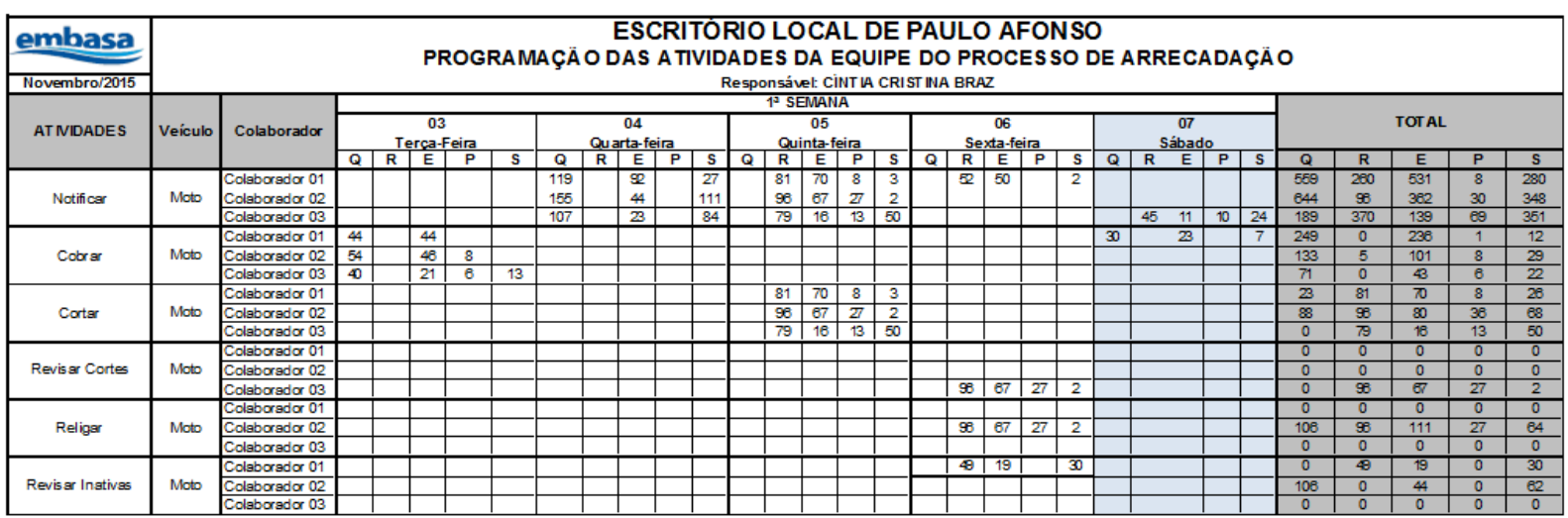

Legenda: $\mathrm{Q}=$ Quantidade $. \mathrm{R}=$ Reprogramado. $\mathrm{E}=$ Executados. $\mathrm{P}=$ Pagos. $\mathrm{S}=$ Sobras.

\begin{tabular}{|c|c|c|c|c|c|c|c|}
\hline \multirow{2}{*}{ ATIVIDADES } & \multirow{2}{*}{ Veículo } & \multirow{2}{*}{ Colaborador } & \multicolumn{5}{|c|}{ TOTAL } \\
\hline & & & $Q$ & $\mathbf{R}$ & $\mathbf{E}$ & $\mathbf{P}$ & $S$ \\
\hline \multirow{3}{*}{ Notificar } & \multirow{3}{*}{ Moto } & Colaborador 01 & 559 & 260 & 531 & 8 & 280 \\
\hline & & Colaborador 02 & 644 & 150 & 415 & 31 & 348 \\
\hline & & Colaborador 03 & 189 & 419 & 158 & 69 & 381 \\
\hline \multirow{3}{*}{ Cobrar } & \multirow{3}{*}{ Moto } & Colaborador 01 & 249 & 0 & 236 & 1 & 12 \\
\hline & & Colaborador 02 & 133 & 5 & 101 & 8 & 29 \\
\hline & & Colaborador 03 & 71 & 0 & 43 & 6 & 22 \\
\hline \multirow{3}{*}{ Cortar } & \multirow{3}{*}{ Moto } & Colaborador 01 & 23 & 0 & 0 & 0 & 23 \\
\hline & & Colaborador 02 & 88 & 0 & 13 & 9 & 66 \\
\hline & & Colaborador 03 & 0 & 0 & 0 & 0 & 0 \\
\hline \multirow{3}{*}{ Revisar Cortes } & \multirow{3}{*}{ Moto } & Colaborador 01 & 0 & 0 & 0 & 0 & 0 \\
\hline & & Colaborador 02 & 0 & 0 & 0 & 0 & 0 \\
\hline & & Colaborador 03 & 0 & 0 & 0 & 0 & 0 \\
\hline \multirow{3}{*}{ Religar } & \multirow{3}{*}{ Moto } & Colaborador 01 & 0 & 0 & 0 & 0 & 0 \\
\hline & & Colaborador 02 & 106 & 0 & 44 & 0 & 62 \\
\hline & & Colaborador 03 & 0 & 0 & 0 & 0 & 0 \\
\hline \multirow{3}{*}{ Revisar Inativas } & \multirow{3}{*}{ Moto } & Colaborador 01 & 23 & 0 & 0 & 0 & 23 \\
\hline & & Colaborador 02 & 88 & 0 & 13 & 9 & 66 \\
\hline & & Colaborador 03 & 0 & 0 & 0 & 0 & 0 \\
\hline
\end{tabular}

Figura 02: Layout da função de programação semanal.

Fonte: Ferramenta de gerenciamento de rotina e produtividade.

- Mensurar a produtividade da equipe: 
Id on Line Revista Multidisciplinar e de Psicologia

Id on Line Multidisciplinary and Psychology Journal

Figura 03: Layout da função de mensuração da produtividade.

Fonte: Ferramenta de gerenciamento de rotina e produtividade.

- Analisar a eficiência na execução das atividades:

\begin{tabular}{|l|c|c|c|c|c|}
\hline \multicolumn{7}{|c|}{ EFICIÊNCIA NA EXECUÇÃO DOS SERVIÇOS } \\
\hline \multicolumn{1}{|c|}{ Atividades } & Quantidade & Reprogramados & Executados & Pagos & Sobras \\
\hline Notificações & 6.946 & 500 & 5.865 & 100 & 1.481 \\
\hline Cobranças & 1.143 & 100 & 1.083 & 41 & 119 \\
\hline Cortes & 267 & 0 & 105 & 90 & 72 \\
\hline Revisões de Cortes & 773 & 50 & 737 & 0 & 86 \\
\hline Religações & 215 & 0 & 215 & 0 & 0 \\
\hline Revisões de Inativas & 106 & 0 & 102 & 0 & 4 \\
\hline \multicolumn{1}{|c|}{ TOTAL } & $\mathbf{9 . 4 5 0}$ & $\mathbf{6 5 0}$ & $\mathbf{8 . 1 0 7}$ & $\mathbf{2 3 1}$ & $\mathbf{1 . 7 6 2}$ \\
\hline
\end{tabular}

EFICIÊNCIANA EXECUÇÃO DOS SERVIÇOS

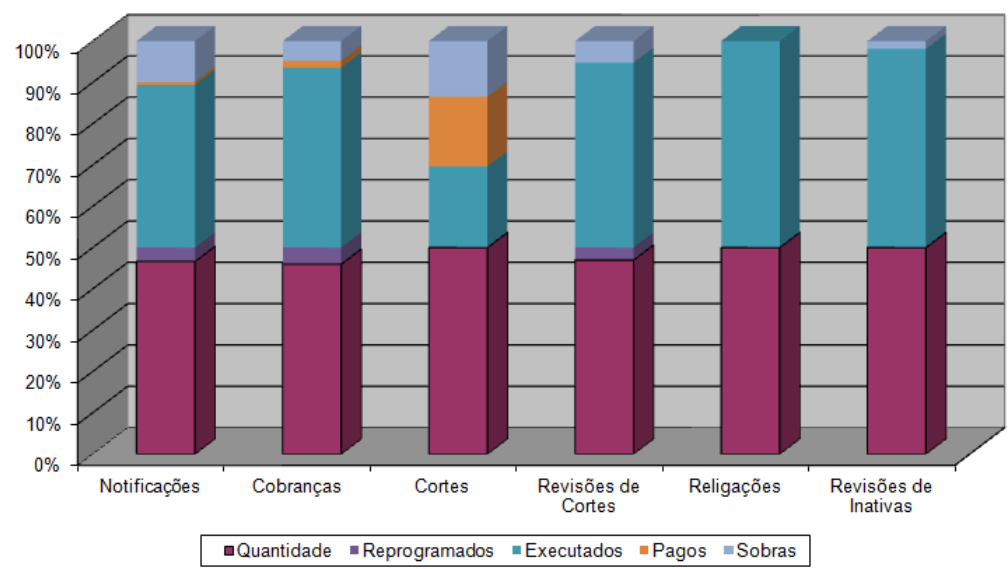

Figura 04: Layout da função de mensuração da eficiência na execução dos serviços.

Fonte: Ferramenta de gerenciamento de rotina e produtividade.

- $\quad$ Analisar o custo Equipe x Serviço:

\begin{tabular}{|l|c|c|c|c|c|}
\hline \multicolumn{7}{|c|}{ EFICIÉNCIA NA EXECUÇÃO DOS SERVIÇOS } \\
\hline \multicolumn{1}{|c|}{ Atividades } & Quantidade & Reprogramados & Executados & Pagos & Sobras \\
\hline Notificaç̃os & 6.946 & 500 & 5.865 & 100 & 1.481 \\
\hline Cobranças & 1.143 & 100 & 1.083 & 41 & 119 \\
\hline Cortes & 267 & 0 & 105 & 90 & 72 \\
\hline Revisões de Cortes & 773 & 50 & 737 & 0 & 86 \\
\hline Religações & 215 & 0 & 215 & 0 & 0 \\
\hline Revisões de Inativas & 106 & 0 & 102 & 0 & 4 \\
\hline \multicolumn{1}{|c|}{ TOTAL } & 9.450 & 650 & $\mathbf{8 . 1 0 7}$ & 231 & $\mathbf{1 . 7 6 2}$ \\
\hline
\end{tabular}

\begin{tabular}{|c|c|c|}
\hline \multicolumn{3}{|c|}{ VALOR DOS SERVIÇOS NO CONTRATO } \\
\hline Valor Serviço & Valor Total & Valor Visitas \\
\hline $\mathrm{R} \$ 0,44$ & $\mathrm{R} \$ 2.580,60$ & $\mathrm{R} \$ 57,00$ \\
\hline $\mathrm{R} \$ 4,55$ & $\mathrm{R} \$ 4.927,65$ & $\mathrm{R} \$ 23,37$ \\
\hline $\mathrm{R} \$ 8,12$ & $\mathrm{R} \$ 852,60$ & $\mathrm{R} \$ 51,30$ \\
\hline $\mathrm{R} \$ 4,55$ & $\mathrm{R} \$ 3.353,35$ & $\mathrm{R} \$ 0,00$ \\
\hline $\mathrm{R} \$ 9,20$ & $\mathrm{R} \$ 1.978,00$ & $\mathrm{R} \$ 0,00$ \\
\hline $\mathrm{R} \$ 13,11$ & $\mathrm{R} \$ 1.337,22$ & $\mathrm{R} \$ 0,00$ \\
\hline TOTAL & $\mathrm{R} \$ 15.029,42$ & $\mathrm{R} \$ 131,67$ \\
\hline
\end{tabular}

\begin{tabular}{|l|c|c|c|}
\hline \multicolumn{4}{|c|}{ CUSTO DA EQUIPE NO CONTRATO } \\
\hline \multicolumn{1}{|c|}{ Item Contrato } & Valor Unitário & Quantidade & Valor Total \\
\hline Ag. Serviço Comercial & $\mathrm{R} \$ 2.051,93$ & 6 & $\mathrm{R} \$ 12.311,58$ \\
\hline Moto & $\mathrm{R} \$ 702,20$ & 5 & $\mathrm{R} \$ 3.511,00$ \\
\hline \multicolumn{4}{|c|}{ TOTAL } \\
\hline
\end{tabular}

\begin{tabular}{l|r} 
Relação Serviços x Equipe Fixa & $4,18 \%$ \\
\hline
\end{tabular} 
Id on Line Revista Multidisciplinar e de Psicologia

Id on Line Multidisciplinary and Psychology Journal

Figura 05: Layout da função de medição de custos.

Fonte: Ferramenta de gerenciamento de rotina e produtividade.

As fases de monitoramento e controle fazem parte da rotina do Processo, sendo executadas diariamente pela liderança do Processo com o auxílio da ferramenta. A partir dos resultados mensais é possível ajustar as ações para melhorar um ponto crítico apresentado anteriormente.

A Ferramenta Gerenciamento de Rotina e Produtividade no Processo de Arrecadação do EL de Paulo Afonso foi implementada em julho de 2013 e os resultados apresentados após o início de sua utilização podem ser visualizados nos gráficos a seguir:

- Reflexo na Arrecadação da Cidade:

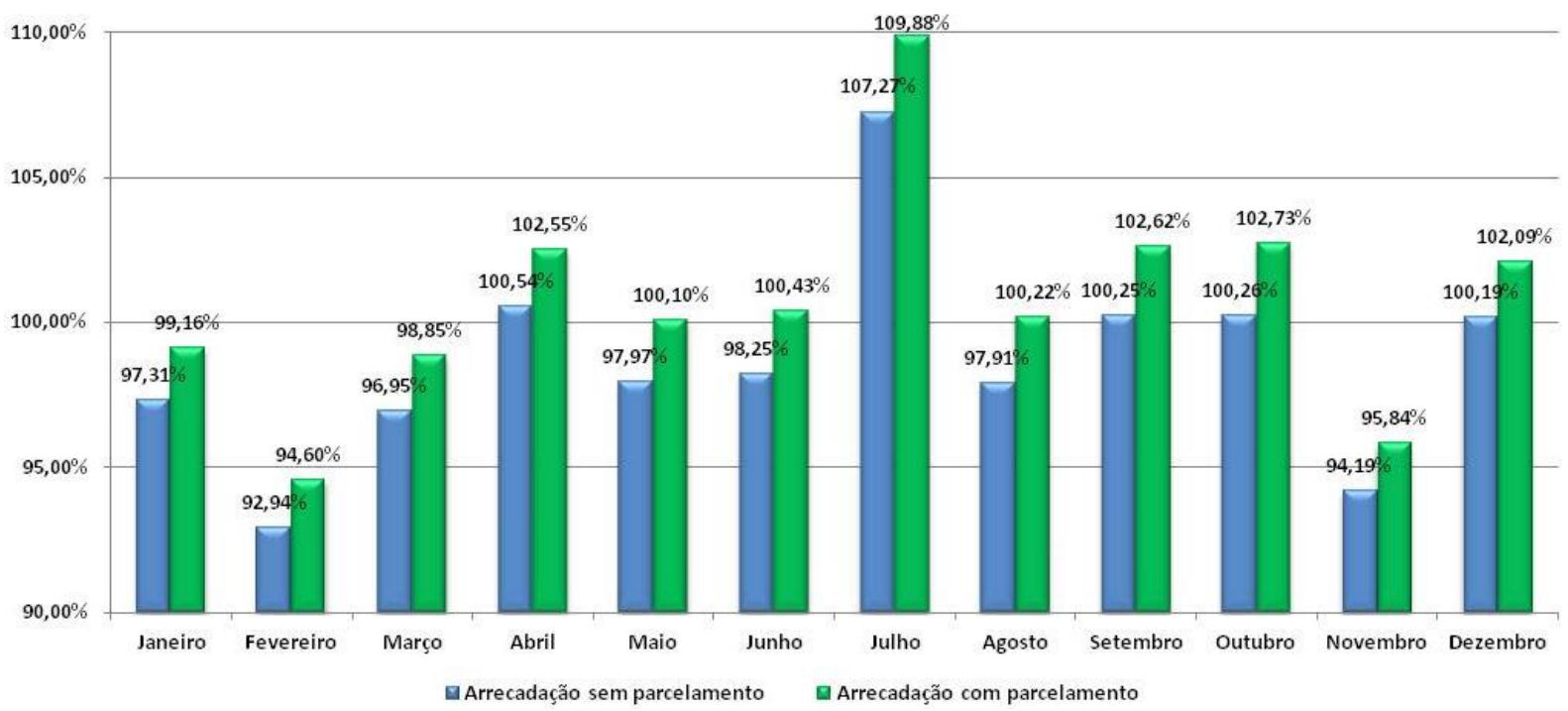

Gráfico 01: Eficiência na Arrecadação Particular da Cidade de Paulo Afonso em 2013.

Fonte: Relatório Gerencial SCI/WEB.

O gráfico 01 demonstra o reflexo do gerenciamento de rotina e produtividade na eficiência da arrecadação particular na cidade de Paulo Afonso, destacando 9,88\% acima de $100 \%$ no primeiro mês (julho) de implantação da ferramenta e sua manutenção superior a 100\% nos meses seguintes.

- $\quad$ Reflexo na Arrecadação da UNP: 
on Line Revista Multidisciplinar e de Psicologia

Id on Line Multidisciplinary and Psychology Journal

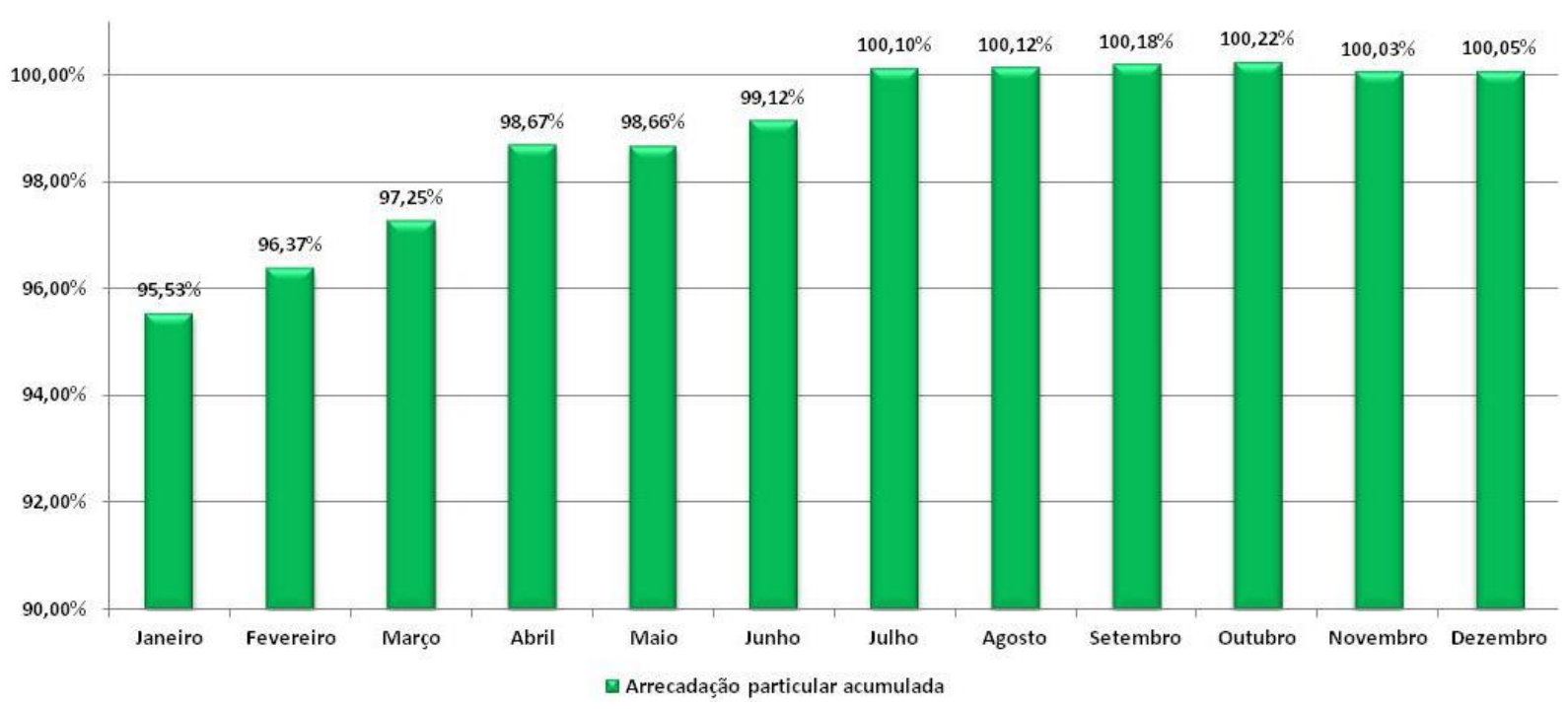

Gráfico 02: Eficiência na Arrecadação Particular Acumulada da UNP em 2013.

Fonte: Relatório Gerencial SCI/WEB.

O gráfico 02 demonstra o reflexo do gerenciamento de rotina e produtividade na eficiência da arrecadação particular da unidade regional de Paulo Afonso, destacando porcentagens acima de 100\% em todos os meses seguintes à implantação da ferramenta (julho).

\section{Considerações Finais}

O gerenciamento da rotina é uma atividade complementar ao processo de melhoria na gestão, pois auxilia no monitoramento e controle da execução diária e interação das atividades dos processos de trabalho.

A abordagem por processos permite melhor especificação do trabalho realizado, o desenvolvimento de sistemas, a gestão do conhecimento, o redesenho e a melhoria, por meio da análise do trabalho realizado de modo a identificar oportunidades de aperfeiçoamento (BRASIL: 2013, p. 13).

Os benefícios agregados a Embasa a partir da criação e implementação da Ferramenta de Gerenciamento de Rotina e Produtividade no Processo de Arrecadação do EL de Paulo Afonso vão desde a elevação da produtividade e eficiência dos resultados até a garantia da qualidade do trabalho realizado e das entregas produzidas.

As ações apresentadas neste estudo evidenciam a concepção da Embasa quanto à importância da Gestão por Processos para o aprimoramento da gestão, de forma que a aplicação das etapas de gerenciamento de suas rotinas estimula os atores a produzirem e analisarem informações gerenciais e a pensarem e discutirem ações de melhorias dos processos nos quais estão inseridas. 


\section{Referências}

BARROS, Aidil de Jesus Paes de \& LEHFELD, Neide Aparecida de Souza. Projeto de Pesquisa: propostas metodológicas. 13 ed. Petrópolis, RJ: Vozes, 2002.

BEST, J. N. Como investigar en educación. 2 ed. Madrid: Morata, 1972.

BRASIL. Ministério do Planejamento, Orçamento e Gestão. Secretaria de Logística e Tecnologia da Informação. Departamento de Governo Eletrônico. Guia de gestão de processos de governo. Brasília, 2011, 93p. Disponível em: <http://www.gespublica.gov.br/Tecnologias/pasta.2010-04$26.0851676103 /$ Guia\%20 de\%20Gestao\%20de\%20Processos\%20de\%20Governo.pdf $>$. Acesso em: 20 jan. 2016.

. Ministério do Planejamento, Orçamento e Gestão. Secretaria de Gestão. Programa Nacional de Gestão Pública e Desburocratização - GESPÚBLICA; Prêmio Nacional da Gestão Pública - PQGF. Carta de Serviços ao Cidadão. Brasília: MPOG, Seges, 2009. Disponível em: <http://www.gespublica.gov.br/biblioteca/pasta. 2013-0328.9924370611/Carta\%20de\%20Servicos\%20ao\%20Cidadao\%20-\%20guia \%20metodologico.pdf $>$. Acesso em: 27 jan. 2016.

Ministério do Planejamento, Orçamento e Gestão. Secretaria de Gestão Pública. Programa GESPÚBLICA. Modelo de Excelência em Gestão Pública. Brasília: MP/SEGEP, 2014. Disponível em: $\quad<$ http://www.gespublica.gov.br/pasta. 2014-0612.8013949452/Modelo\%20de\%20Excelencia\%20em\%20Gestao\%20Publi ca\%202014\%20vs\%2005062014.pdf>. Acesso em: 27 jan. 2016.

Procuradoria Geral da República. Secretaria Jurídica e de Documentação. Manual de gestão por processos. Brasília: MPF/PGR, 2013. Disponível em: <http://www.mpf.mp.br/conheca-o$\mathrm{mpf} / \mathrm{gestao}$-estrategica-e-modernizacao-do-mpf/es critorio-deprocessos/publicacoes/livros/manualdegestaoporprocessos.pdf>. Acesso em: 20 jan. 2016.

CAMPOS, Vicente Falconi. TQC: Controle da qualidade total. Rio de Janeiro: Bloch, 1992. CAPOTE, Gart. Guia para Formação de Analistas de Processos - BPM. 1. ed. Rio de Janeiro, 2011.

EMBASA. Manual de Cobrança de Débitos. Salvador: Superintendência Comercial, 2012.

Relatório Gerencial SCI/WEB. Salvador: Superintendência Comercial, 2013.

GOLDENBERG, Mirian. A arte de pesquisar: como fazer pesquisa qualitativa em Ciências Sociais. 7 ed. Rio de Janeiro: Record, 2003.

LAKATOS, Eva Maria. Metodologia científica: Ciência e conhecimento científico, métodos científicos, teoria, hipóteses e variáveis. São Paulo: Atlas, 1986.

MOURA, Luciano Raizer. Qualidade simplesmente total: uma abordagem simples e prática da gestão da qualidade. Rio de Janeiro: Qualitymark, 1997.

NORMANN, Richard. Administração de serviços: estratégia e liderança na empresa de serviços. São 
Id on Line Revista Multidisciplinar e de Psicologia

Id on Line Multidisciplinary and Psychology Journal

Paulo: Atlas, 1993.

PAIM, R; CARDOSO, V; CAULLIRAUX, H; CLEMENTE, R. Gestão de Processos:

Pensar, Agir e Aprender. São Paulo: Editora Artmed, 2009.

SANTOS, Edilene Galdino dos; LIMA, Izabel França de; ABRANTES, Mônica Paiva Santos de. Gerenciamento da rotina diária em unidades de informação. Disponível em: <http://dici.ibict.br/archive/00000714/01/T054>. Acesso em: 14 fev. 2016.

SELLTIZ, C. et al. Métodos de pesquisa nas relações sociais. São Paulo: Herder, 1965.

\section{Como citar este artigo (Formato ABNT):}

BRÁZ, C.C.; QUEVEDO, A.P.F.; CAFFÉ FILHO, H.P. Implementação de uma Ferramenta de Gerenciamento de Rontina e produtividade. Id on Line Revista Multidisciplinar e de Psicologia, Julho de 2016, vol.10, n.30. Supl. 1, p. 78-91. ISSN 1981-1179.

Recebido: 28/04/2016

Aceito: 02/05/2016 